Published in final edited form as:

Biomacromolecules. 2016 August 08; 17(8): 2507-2513. doi:10.1021/acs.biomac.6b00350.

\title{
Hypoxia-Responsive Polymersomes for Drug Delivery to Hypoxic Pancreatic Cancer Cells
}

\author{
Prajakta Kulkarni ${ }^{\dagger}$, Manas K. Haldar ${ }^{\dagger}$, Seungyong You ${ }^{\ddagger}$, Yongki Choi ${ }^{\ddagger}$, and Sanku Mallik ${ }^{*}, \ddagger$ \\ tDepartment of Pharmaceutical Sciences, North Dakota State University, Fargo, North Dakota \\ 58102, United States \\ ¥Department of Physics and Mathematics, North Dakota State University, Fargo, North Dakota \\ 58102, United States
}

\begin{abstract}
Hypoxia in tumors contributes to overall tumor progression by assisting in epithelial-tomesenchymal transition, angiogenesis, and metastasis of cancer. In this study, we have synthesized a hypoxia-responsive, diblock copolymer poly(lactic acid)-azobenzene-poly(ethylene glycol), which self-assembles to form polymersomes in an aqueous medium. The polymersomes did not release any encapsulated contents for 50 min under normoxic conditions. However, under hypoxia, $90 \%$ of the encapsulated dye was released in $50 \mathrm{~min}$. The polymersomes encapsulated the combination of anticancer drugs gemcitabine and erlotinib with entrapment efficiency of $40 \%$ and $28 \%$, respectively. We used three-dimensional spheroid cultures of pancreatic cancer cells BxPC-3 to demonstrate hypoxia-mediated release of the drugs from the polymersomes. The vesicles were nontoxic. However, a significant decrease in cell viability was observed in hypoxic spheroidal cultures of BxPC-3 cells in the presence of drug encapsulated polymersomes. These polymersomes have potential for future applications in imaging and treatment of hypoxic tumors.
\end{abstract}

\section{Abstract}

*Corresponding Author. Sanku Mallik. Phone: 701-231-7888. Fax: 701-231-7831. Sanku.Mallik@ndsu.edu. ASSOCIATED CONTENT

Supporting Information

The Supporting Information is available free of charge on the ACS Publications website at DOI: 10.1021/acs.biomac.6b00350.

Hypoxia-responsive characteristic of azobenzene linker, analysis of hypoxia-responsive polymer by ${ }^{1} \mathrm{H}$ NMR spectroscopy, GPC of synthesized hypoxia-responsive polymersomes, cellular uptake for control polymersomes under normoxic and hypoxic conditions, laser scanning confocal imaging for stained polymersome vesicles, and UV spectra of gemcitabine, erlotinib, and the hypoxiaresponsive polymer (PDF)

Author Contributions

The manuscript was written through contributions of all authors. All authors have given approval to the final version of the manuscript. 


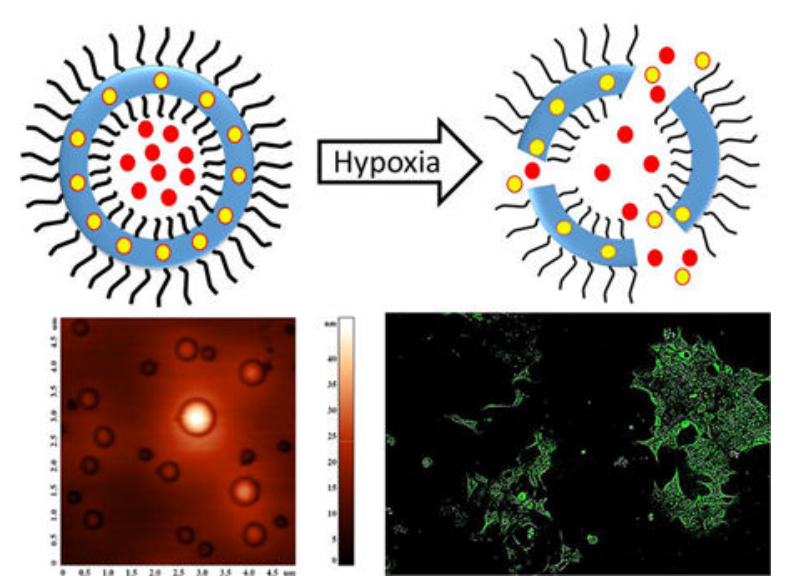

\section{INTRODUCTION}

Rapid cell division, exponential growth, and insufficient blood supply create oxygen gradients in solid tumors. ${ }^{1}$ Oxygen partial pressure in the tumors decreases from the surface to the interior, reaching as low as $0-2.5 \mathrm{mmHg}$ in some regions. ${ }^{2}$ These hypoxic areas assist disease progression by remodeling the extracellular matrix, initiating the epithelial-tomesenchymal transition, and altering the overall biochemical environment around the cells. ${ }^{3}$ Hypoxia also plays a significant role in developing resistance to radio and chemotherapy in cancer patients. ${ }^{4}$

Hypoxia develops in solid tumors of breast, colon, prostate, and pancreatic cancers. ${ }^{5}$ This problem is exacerbated in pancreatic cancer due to formation of dense extracellular matrix (desmoplasia) and early development of hypoxia. ${ }^{7}$ The hypoxic regions and desmoplasia make treatment ineffective for pancreatic cancer, leading to a dismal five-year survival rate of about $5 \% .^{6-8}$

Hypoxic and normoxic tissues show remarkably different microenvironments, providing an opportunity for tumor-specific drug delivery with reduced oxygen partial pressure as the trigger. ${ }^{9}$ Polymersomes are vesicles formed from amphiphilic diblock copolymers capable of encapsulating hydrophilic compounds in the core and hydrophobic drugs in the bilayer. ${ }^{10}$ The relative ratio of the hydrophobic and hydrophilic polymer blocks determines the formation of polymersomes. ${ }^{10}$ The reported tumor-targeted polymersomes deliver the encapsulated drugs at the targeted site in response to the elevated levels of enzymes, reducing agents, reduced $\mathrm{pH}$, etc. ${ }^{11}$ However, hypoxia-responsive polymeric drug carriers are less explored. Polymeric nanoparticles with the nitroimidazole pendant groups released encapsulated doxorubicin in a hypoxic environment. ${ }^{12}$ The reducible azobenzene group has been used to prepare imaging agents and polymeric micelles responsive to the reducing microenvironment of hypoxia. ${ }^{13,14}$

In this study, we have synthesized a hypoxia-responsive, amphiphilic diblock copolymer by conjugating poly(lactic acid) (PLA) with poly(ethylene glycol) (PEG) via an azobenzene linker. We prepared polymersomes from the synthesized copolymer, encapsulating the anticancer drug gemcitabine and the epidermal growth factor receptor (EGFR) inhibitor 
erlotinib. Gemcitabine is the first choice anticancer drug for pancreatic cancer. ${ }^{15}$ The EGFR receptor inhibitors aid in restricting the disease progression. ${ }^{16}$ Clinical trials indicate improved survival of pancreatic cancer patients when gemcitabine is combined with erlotinib. ${ }^{8}$ However, erlotinib is more hydrophobic compared with gemcitabine. Hence, encapsulation of both drugs in polymersomes has the potential to increase the overall effectiveness of the treatment. We expected that hypoxic conditions will reduce the azobenzene group of the polymers to amines. ${ }^{14}$ Herein, we demonstrate that the resultant destabilization of the polymer bilayer releases the encapsulated drugs from the polymersomes to cultured hypoxic spheroids of pancreatic cancer cells BxPC-3.

\section{MATERIALS AND METHODS}

\section{Synthesis and Characterization of the Copolymer}

Reaction of PEG-Diphenylazacarboxylate with 1- Aminopropanol-Polymer m$\mathrm{PEG}_{1900}$-amine was conjugated to azobenzene- $4,4^{\prime}$-dicarboxylic acid by following a previously published protocol. ${ }^{13}$ The PEG-diphenylazacarboxylate $(1 \mathrm{~g}, 0.46 \mathrm{mmol})$ was dissolved in pyridine $(25 \mathrm{~mL})$. To this solution, 1-ethyl-3-(3-dimethylaminopropyl)carbodiimide (EDC; $134 \mathrm{mg}, 0.69 \mathrm{mmol}$ ) and $N$-hydroxysuccinimide (NHS; $80 \mathrm{mg}, 0.69$ $\mathrm{mmol}$ ) were added followed by excess 1 - aminopropanol $(175 \mathrm{mg}, 2.32 \mathrm{mmol})$. The reaction mixture was stirred overnight. The solvent was then removed under reduced pressure. The residue obtained was dissolved in dichloromethane and washed with $0.5 \mathrm{~N} \mathrm{HCl}$ and $0.5 \mathrm{~N}$ $\mathrm{NaOH}$. The clear organic solution was dried over $\mathrm{Na}_{2} \mathrm{SO}_{4}$, evaporated, and subjected to chromatographic purification $\left(R_{f}=0.6,10 \% \mathrm{MeOH}\right.$ in dichloromethane) yielding $677 \mathrm{mg}$ $(66 \%)$ of the yellow semisolid product. ${ }^{1} \mathrm{H}$ NMR ( $400 \mathrm{MHz}$, chloroform- $d$ ) $\delta$ ppm: 0.02 $0.01(\mathrm{~m}), 1.08-1.36(\mathrm{~m}), 3.37-3.38(\mathrm{~m}), 3.53-3.63(\mathrm{~m}), 3.77-3.91(\mathrm{~m}), 5.25-5.38(\mathrm{~m}), 7.27$ (s), 7.93-8.07 (m).

Synthesis of the Block Copolymer-The product obtained from the previous step (150 $\mathrm{mg}, 0.068 \mathrm{mmol}$ ) was taken into toluene $(30 \mathrm{~mL})$ and was subjected to azeotropic distillation for $6 \mathrm{~h}$ employing a Dean-Stark apparatus. After the solution was cooled to room temperature under nitrogen, D,L-lactide $(450 \mathrm{mg}, 3.17 \mathrm{mmol}$ ) and tin(II) ethoxyhexanoate $(15 \mathrm{mg})$ were added, and the solution was heated to reflux under nitrogen for $14 \mathrm{~h}$. After cooling to room temperature, the reaction mixture was added dropwise to the cold ether. After $3 \mathrm{~h}$, the clear supernatant was decanted, and the precipitate was dried under vacuum. The yellow viscous semisolid product obtained (405 mg, 67\%) was analyzed by ${ }^{1} \mathrm{H}$ NMR spectroscopy and gel permeation chromatography for purity and molecular weight (Supporting Information, Figures S2 and S3). ${ }^{1} \mathrm{H}$ NMR (400 MHz, chloroform- $d$ ) $\delta \mathrm{ppm}$ : 0.00-0.11 (m), 1.40-1.61 (m), 1.68-1.78 (m), 1.81-2.11 (m), 3.62-3.89 (m), 3.92-4.16 (m), $5.08-5.35(\mathrm{~m})$.

\section{Preparation of Polymersomes Encapsulating Carboxyfluorescein Dye}

The synthesized hypoxia-responsive polymer $\mathrm{PLA}_{80}$-azobenzene- $\mathrm{PEG}_{47}$ was dissolved in tetrahydrofuran $(10 \mathrm{mg} / \mathrm{mL})$, and the solution $(200 \mu \mathrm{L})$ was added dropwise to a stirred solution ( $2 \mathrm{~mL}$ ) of carboxyfluorescein dye (100 mM) in HEPES buffer ( $25 \mathrm{mM}, \mathrm{pH} 7.4)$. After stirring for $1 \mathrm{~h}$, the THF was evaporated by bubbling air through the solution to form 
the carboxyfluorescein encapsulated polymersomes. The formed polymersomes were then sonicated for $1 \mathrm{~h}$ in a bath sonicator (Aquasonic 250D, level 9), and dialyzed (molecular cutoff 1000) overnight in iso-osmolar HEPES buffer $(25 \mathrm{mM}, \mathrm{pH} 7.4)$ to remove unencapsulated carboxyfluorescein. The polymersomes were then passed through Sephadex G100 gel filtration column to remove the remaining unencapsulated carboxyfluorescein. The polymersomes collected were used for the release and cellular uptake studies.

\section{Preparation of Polymersomes Encapsulating Gemcitabine and Erlotinib}

The synthesized polymer PLA 80 -azobenzene- $\mathrm{PEG}_{47}$ was dissolved in tetrahydrofuran (10 $\mathrm{mg} / \mathrm{mL}$ ) and a fluorescent lipid, 1,2-dipalmitoyl-sn-glycero-3-phosphoethanolamine- $N$ lissamine rhodamine B sulfonyl ammonium salt (rhodamine lipid) was dissolved in chloroform $(0.01 \mathrm{mg} / \mathrm{mL})$. To an empty vial, rhodamine lipid solution was added, and chloroform was evaporated from the bottle. To the same vial, the polymer solution ( $200 \mu \mathrm{L}$, $2 \mathrm{mg}$ ) was added to adjust the polymer to lipid molar ratio to 95:5. The solvent was evaporated from the vial by passing air through it, and $200 \mu \mathrm{L}$ of THF was added to make the concentration of polymer $10 \mathrm{mg} / \mathrm{mL}$. In another vial, erlotinib was dissolved in $200 \mathrm{mM}$ citrate buffer ( $\mathrm{pH} 4$ ) to make drug concentration $0.2 \mathrm{mg} / \mathrm{mL}$. Polymers dissolved in THF were then added dropwise to an erlotinib-citrate buffer solution $(2 \mathrm{~mL})$. The mixture was stirred for $1 \mathrm{~h}$ at room temperature. THF was evaporated by passing air through the mixture for $45 \mathrm{~min}$. The polymersomes formed were then sonicated using a bath sonicator (Aquasonic model 250D, level 9) for $45 \mathrm{~min}$, and the polymersomes were passed through SephadexG-100 gel filtration column saturated with HEPES buffer ( $25 \mathrm{mM}, \mathrm{pH}$ 7.4). This protocol generated a $\mathrm{pH}$ gradient across the polymersome membrane (surrounding $\mathrm{pH} 7.4$, core $\mathrm{pH} 4$ ). Gemcitabine was added to these polymersomes (polymer to drug ratio 5:1 by weight), and the resulting mixture was stirred for $4 \mathrm{~h}$ at room temperature. The polymersomes were again passed through the SephadexG-100 gel filtration column to remove unencapsulated drugs. Drug encapsulation was determined from the UV spectra for the polymersomes ( $270 \mathrm{~nm}$ for gemcitabine and $247 \mathrm{~nm}$ for erlotinib). The amount of drug encapsulated was measured from the standard curves. To calculate entrapment efficiency and loading capacity of the polymersomes, the following equations were used.

percent entrapment efficiency $=\frac{\text { amount of drug added }- \text { amount of drug encapsulated }}{\text { amount of drug added }} \times 100$

$$
\text { percent loading efficiency }=\frac{\text { total weight of drug loaded in polymersomes }}{\text { total weight of polymer }} \times 100
$$

\section{Size Analysis}

The hydrodynamic diameters of polymersomes were measured using a dynamic light scattering (DLS) instrument (Malvern Zetasizer Nano-ZS90). Measurements were conducted at a scattering angle of $90^{\circ}$ using disposable polystyrene cuvette. An equilibration time of $120 \mathrm{~s}$ was maintained for all the measurements. For each sample, six readings were recorded averaging six runs for the same reading. 


\section{Transmission Electron Microscopy}

Copper TEM grids (300-mesh, Formvar-carbon coated, Electron Microscopy Sciences, Hatfield, Pennsylvania, USA) were prepared by applying a drop of $0.01 \%$ poly(L-lysine), allowing it to stand for $30 \mathrm{~s}$, wicking off the liquid with torn filter paper, and allowing the grids to air-dry. A drop of the suspension diluted 1:100 was placed on a prepared grid for 30 $\mathrm{s}$ and wicked off; grids were allowed to air-dry again. Phosphotungstic acid, $0.1 \% \mathrm{pH}$ adjusted to 7-8, was dropped onto the grid containing the sample, allowed to stand for 2 min, and wicked off. After the grids had dried, images were obtained using a JEOL JEM-2100 LaB6 transmission electron microscope (JEOL USA, Peabody, Massachusetts) running at $200 \mathrm{keV}$.

\section{Release Studies}

Carboxyfluorescein encapsulated polymersomes $(200 \mu \mathrm{L})$ were added to $1.6 \mathrm{~mL}$ of isoosmolar HEPES buffer ( $25 \mathrm{mM}$, pH 7.4). To this solution, $100 \mu \mathrm{L}$ of rat liver microsomes were added along with $100 \mu \mathrm{M}$ NADPH. The rat liver microsomes were isolated using a reported protocol. ${ }^{17}$ Air was bubbled through the reaction mixture to create normoxic conditions. For hypoxic conditions, nitrogen gas was bubbled through the reaction mixture. The emission intensity was recorded every $5 \mathrm{~min}$ for $1 \mathrm{~h}$ using a spectrofluorimeter at the emission wavelength of $515 \mathrm{~nm}$ (excitation $480 \mathrm{~nm}$ ). After $1 \mathrm{~h}$ of normoxic or hypoxic treatment, $20 \mu \mathrm{L}$ of Triton was added to disintegrate the polymersomes and release the encapsulated dye. Fluorescence intensity was measured for the total release after complete disintegration. Percent release was calculated using following formula:

$$
\frac{(\text { emission intensity after release }- \text { intensity before release })}{\text { (intensity after Triton treatment }- \text { intensity before release) }} \times 100
$$

Cumulative percent release profile was plotted as a function of time.

\section{Atomic Force Microscopic (AFM) Imaging}

A drop of polymersome solution was placed on top of a freshly cleaved mica surface. The surface was evenly coated with the polymersomes using spin coater at $2000 \mathrm{rpm}$ and then dried. The AFM imaging was carried out in non-contact mode at a scanning rate of $0.7 \mathrm{~Hz}$ and a resonance frequency of $145 \mathrm{kHz}$ using an NT-MDT NTEGRA (NT-MDT America, Tempe, AZ). The mica substrate was glued on top of a sapphire substrate, which is a sample holder, using Scotch double sided tape and cleaved with Scotch tape to obtain a debris-free and flat surface. The cantilever was made of silicon nitride and was $100 \mu \mathrm{m}$ long. The scanning areas were $5 \times 5$ or $20 \times 20 \mu \mathrm{m}^{2}$ at the resolution of 512 or 1024 points per line, respectively. The images were flattened by a first order line correction and a first order plane subtraction to compensate a sample tilt.

\section{Cell Culture}

The pancreatic cancer cell line BxPC-3 was purchased from American Type Culture Collection (Manassas, VA). The BxPC-3 cells were cultured in RPMI media (Hyclone) supplemented with $10 \%$ fetal bovine serum (Gibco) and $2 \%$ antibiotics (Corning). The cells 
were grown at $37{ }^{\circ} \mathrm{C}$ in a humidified atmosphere containing $5 \% \mathrm{CO}_{2}$ for normoxic conditions. Hypoxia was induced in the cells by culturing in Biospherix $\mathrm{C} 21$ hypoxic chamber supplemented with $1 \%$ oxygen.

\section{Cell Viability Assay}

Cytotoxicity of the gemcitabine and erlotinib encapsulated polymersomes was tested on BxPC-3 cells. The cells were incubated (2000 per well) in a 96-well sterile plate. RPMI media supplemented with $10 \%$ FBS was added to each well $(200 \mu \mathrm{L})$, and the cells were allowed to grow for one doubling time. The plate was divided into three groups: control, drug treated, and test polymersomes treated. The control polymersomes (Control P) were prepared using $\mathrm{PLLA}_{5000}-\mathrm{PEG}_{2000}$ polymer (Polyscitech). This polymer has similar chain length for hydrophilic and hydrophobic portions as observed in the synthesized polymer and can act as a good control in hypoxic conditions due to its nonresponsiveness to hypoxic environment. Six replicates were recorded for each sample. The control group received buffer encapsulated polymersomes. Polymersomes prepared for the cellular studies were prepared in a buffer iso-osmolar with the cell culture medium to avoid premature leakage from the polymeric vesicles. Drug-treated cells received the gemcitabine $(20 \mu \mathrm{M})$-erlotinib $(7.5 \pm 1 \mu \mathrm{M})$ solution equivalent to the encapsulated drug concentration. Test polymersome treated cells received an equivalent amount of encapsulated gemcitabine $(20 \mu \mathrm{M})$ and erlotinib $(7.5 \pm 1 \mu \mathrm{M})$. The cells were treated for 3 days, and cell toxicity was recorded after $72 \mathrm{~h}$ with the Alamar Blue assay by following the supplier's (Life Technologies) protocol. Alamar Blue solution $(10 \mu \mathrm{L})$ was added to all the wells and incubated for $2 \mathrm{~h}$. Fluorescence was recorded (excitation/emission wavelength, $585 / 615 \mathrm{~nm}$ ) for cytotoxicity calculation. The viability was determined for the normoxic and the hypoxic BxPC-3 cells.

\section{Cellular Uptake}

The BxPC-3 cells were seeded (5000 per well) on two six well plates. The cells in one plate were allowed to grow in a normoxic environment, and the other was incubated in the hypoxic chamber maintained at $1 \%$ oxygen level. The cells cultured in both the plates were allowed to grow for two doubling times. Cells in both plates were treated with carboxyfluorescein encapsulated polymersomes for 1, 2, and $3 \mathrm{~h}$ under normoxic or hypoxic conditions. The uptake of carboxyfluorescein encapsulated polymersomes and the released dye was observed by fluorescence microscopy. Images were analyzed by ImageJ software.

\section{Cell Viability in Spheroidal Cultures}

The BxPC-3 cells were cultured as three-dimensional spheroids on agarose molds. Briefly, purchased mold (no. 24-35, Microtissues) was used to cast a 35 well agarose scaffold. Scaffolds were seeded with BxPC-3 cells (10 000 cells per scaffold) and were incubated at $37{ }^{\circ} \mathrm{C}$ in a $\mathrm{CO}_{2}$ incubator for 15 days. As the spheroids were formed, the scaffolds were divided into two groups (18 scaffolds in each group). One group was incubated in a normoxic environment (supplemented with $21 \%$ oxygen, $37^{\circ} \mathrm{C}$ ), and the other group was incubated in a hypoxic environment (supplemented with $1 \%$ oxygen, $37^{\circ} \mathrm{C}$ ). After 3 days of incubation, the spheroids were treated with the control polymersomes (encapsulating buffer), gemcitabine and erlotinib combination (concentrations equivalent to test polymersomes), and polymersomes encapsulating gemcitabine and erlotinib (20 $\mu \mathrm{M}$ gemcitabine and $7.5 \pm 1$ 
$\mu \mathrm{M}$ erlotinib). The cells received treatment for 3 days under normoxic or hypoxic conditions. To measure the cell viability after the treatment, the spheroids were dislodged from the scaffolds with centrifugation $\left(1000 \mathrm{~g}, 37^{\circ} \mathrm{C}\right)$ and treated in $15 \mathrm{~mL}$ centrifuge tubes containing $3 \mathrm{~mL}$ of Tryple (recombinant trypsin). The tubes were then incubated at $37{ }^{\circ} \mathrm{C}$ for $20 \mathrm{~min}$ to allow dissociation of attached cells. The cells were then suspended in $10 \mathrm{~mL}$ of culture media and plated on 6 wells of a 96 well plate. Similarly, the cells from all scaffolds were collected and seeded in 96 well plates ( $n=6$ for each group). The cells were allowed to grow in a $\mathrm{CO}_{2}$ incubator at $37^{\circ} \mathrm{C}$ for $24 \mathrm{~h}$. After the treatment, the Alamar Blue assay was carried out following manufacturer's protocol to estimate cell viability for each group.

\section{RESULTS AND DISCUSSION}

Polymersomes are more stable drug carriers compared with micelles and liposomes. The ratio of the hydrophilic and hydrophobic blocks of the amphiphilic polymers is critical for the formation of spherical bilayer vesicles. ${ }^{18}$ We synthesized the azobenzene linked polymer $\mathrm{PLA}_{80}-(\mathrm{AZB})-\mathrm{PEG}_{47}$ (Scheme 1) and characterized it by NMR spectroscopy. The azobenzene group linking the PEG and PLA acts as the hypoxia-responsive unit in the synthesized polymer. ${ }^{19,20}$ The PEG groups on the surface of the polymersomes impart long circulating and passive targeting characteristics. ${ }^{21}$ The amphiphilic nature of the polymersomes allows encapsulation of hydrophilic drugs in the aqueous core and hydrophobic drugs in the membrane. Since the polymer molecules in the bilayer do not flipflop, the structures are considerably more stable compared with liposomes. ${ }^{10,22}$ However, under reducing hypoxic conditions, the azobenzene linker undergoes reduction (mechanism shown in Figure 1) and disrupts the polymer membrane, allowing the release of encapsulated drugs. 14

To ascertain the hypoxia sensitivity, we dissolved the synthesized polymer $(1 \mathrm{mg} / \mathrm{mL})$ in a mixture of tetrahydrofuran and water (1:5) and added the isolated rat liver microsomes (20 $\mu \mathrm{g})$, NADPH $(100 \mu \mathrm{M})$, and bubbled nitrogen gas for $2 \mathrm{~h}$. Subsequently, we filtered the solution and recorded the UV-vis absorption spectrum. We observed a shift in the $\lambda_{\max }$ from 330 to $290 \mathrm{~nm}$ and the emergence of a new band at $230 \mathrm{~nm}$ after exposing the polymer solution to hypoxia (Supporting Information, Figure S1). It is likely that the peak at $230 \mathrm{~nm}$ is due to the formation of the aniline derivatives after the reduction of the azobenzene linker under hypoxia.

The polymersomes were prepared using the synthesized, hypoxia-responsive polymer by the solvent exchange method (Materials and Methods) ${ }^{23}$ and characterized by transmission electron microscopy (TEM, Figure 2A). To ascertain the vesicle formation, we prepared giant polymersomes employing a reported protocol and encapsulated the fluorescent dye FM1-43 in the bilayer (by following a procedure similar to the erlotinib encapsulation). ${ }^{24}$ We imaged the giant vesicles employing a confocal laser scanning microscope. We observed the bilayer of the polymersomes in the images, as reported in the literature (Supporting Information, Figure S5). ${ }^{24}$ The size of the gel-filtered polymersomes was observed to be 83 $\pm 2 \mathrm{~nm}$ with a polydispersity index (PDI) of 0.3 (by dynamic light scattering). A typical size distribution for the polymersomes is shown in Figure 2B. Encapsulation of a dye or the two 
drugs increased the size of the polymersomes to $262 \pm 30 \mathrm{~nm}$. The polydispersity index (PDI) for all the batches was observed to be less than 0.4. (Table1)

To test the stimuli responsiveness of azobenzene incorporated polymersomes, a selfquenching dye (carboxyfluorescein) was encapsulated. Under hypoxia, the polymersomes released $90 \%$ of the encapsulated dye within $50 \mathrm{~min}$ (Figure 3, green squares). No significant release was observed from these vesicles under normoxic conditions (Figure 3, red circles).

Atomic force microscopy indicated that the polymersomes lost their spherical morphology after $1 \mathrm{~h}$ under hypoxia (Figure 4A,B). The treated sample showed irregular shapes, indicating the disintegration of the vesicle structures (Figure 4B). We also observed (by dynamic light scattering) a reduction in the hydrodynamic diameter of the polymersomes (from 90 to $55 \mathrm{~nm}$ ) and an increase in the polydispersity index (from 0.3 to 0.7 ) under hypoxia.

To demonstrate content release in biological systems, we incubated the BxPC-3 cells with the dye-encapsulated polymersomes in a hypoxia chamber supplemented with $1 \%$ oxygen. Although we seeded an equal number of cells in the hypoxic and normoxic (control) plates for imaging, we observed changes in cell morphology in the presence of $1 \%$ oxygen. Hence, we normalized the fluorescence of the images with respect to the number of cells. We analyzed the images by the ImageJ software and calculated the integral density per unit area of the two treatment groups. We noted that the normoxic cells released a small amount of the encapsulated carboxyfluorescein in $3 \mathrm{~h}$ (Figure 5, panel A). However, we observed a significantly higher amount of dye release in the hypoxic cells after $3 \mathrm{~h}$ (Figure 5, panel B).

After confirming the hypoxia-triggered release from the polymersomes, we proceeded to encapsulate the anticancer drugs gemcitabine and erlotinib using a combination of solvent exchange and $\mathrm{pH}$-gradient methods. ${ }^{25,26} \mathrm{Gemcitabine}$ and erlotinib were encapsulated with $40 \% \pm 6 \%$ and $28 \% \pm 8 \%$ entrapment efficiency, respectively. The amount of gemcitabine and erlotinib encapsulated were $100 \pm 15 \mu \mathrm{g}$ and $60 \pm 16 \mu \mathrm{g}$ per mg of the polymer, respectively. For the cellular experiments, the treatment was determined based on the concentration of gemcitabine, because erlotinib is used as an adjuvant to enhance the overall effectiveness of therapy. ${ }^{8}$ The buffer-encapsulated polymersomes showed more than $90 \%$ cell viability in monolayer as well as in spheroidal cultures of the BxPC-3 cells, indicating the nontoxic nature of the drug carrier (Figure 6).

Subsequently, we treated the BxPC-3 cells with drug encapsulated polymersomes for $72 \mathrm{~h}$ (Figure 7A). The unencapsulated gemcitabine and erlotinib and polymersomes devoid of the hypoxia-responsive polymer were used as the controls. In normoxic monolayer cultures, treatment with the gemcitabine and erlotinib resulted in $38 \% \pm 3 \%$ cell viability. Under hypoxic conditions, the cell viability was more $(58 \pm 5 \%)$, showing that the free drugs were less effective than in the normoxic conditions. When the cells were treated with control polymersomes (prepared from PLLA $5000-\mathrm{PEG}_{2000}$ ), the cell viability decreased to $65 \%$ $\pm 4 \%$ under normoxia and $71 \% \pm 3 \%$ under hypoxia. We speculate that the decreased cell viability is a result of slow drug diffusion from the polymersomes to the cell culture media 
in $72 \mathrm{~h}$. Treatment with hypoxia-responsive polymersomes resulted in decreased cell viability, $61 \% \pm 1 \%$ under hypoxia and $77 \% \pm 4 \%$ in normoxia.

Although the monolayer cultures provide valuable information about the cellular function and viability, it does not simulate the three-dimensional architecture of the tumor tissues. To create a better mimic for the tumors, we cultured the BxPC- 3 cells as three-dimensional spheroids in agar molds. ${ }^{27,28}$ Spheroidal cultures contain hypoxic cancer cells in the core as observed in solid tumors. ${ }^{29,30}$ Hence, spheroids are better models for conducting hypoxiaresponsive drug delivery studies in vitro. The 15-day old spheroids were then treated with the polymersomes, analogous to the monolayer cultures (Figure 7B). The results indicated that cell viability was significantly reduced when the spheroids were treated with drug encapsulated in polymersomes. The cell viability under normoxic conditions was unaffected by the hypoxia-responsive polymersomes (Figure 7B, black column, Control). However, the cell viability for the hypoxic spheroids was substantially decreased $(24 \% \pm 4 \%)$ in the presence of the polymersome encapsulated drugs compared with the free drugs $(48 \% \pm 2 \%)$. We observed that the cell viability was significantly reduced in spheroidal cultures compared with the monolayer studies (compare Figure 7, panels A and B). The drug-containing, hypoxia-responsive polymersomes were more effective compared with the free drug combination. We speculate that this difference may be due to the release of drugs in the hypoxic core of the spheroids. ${ }^{29,30}$

\section{CONCLUSION}

In conclusion, our synthesized azobenzene incorporated, amphiphilic PLA-PEG polymer self-assembled into polymeric vesicles. The polymersomes encapsulated the anticancer drugs gemcitabine and erlotinib with loading efficacy of $40 \% \pm 6 \%$ and $28 \% \pm 8 \%$ respectively. These polymersomes successfully released the encapsulated drugs to hypoxic pancreatic cancer cells, resulting in reduced cell viability in both monolayer and spheroidal cultures. Due to the presence of hypoxia-responsive subunit in these polymersomes, the vesicles can be used to image and deliver drugs to the hypoxic regions of tumors.

\section{Acknowledgments}

This research was supported by NSF Grant DMR 1306154 and NIH Grant 1 R01GM 114080 to SM. P.K. was supported by a Doctoral Dissertation Award (IIA-1355466) from the North Dakota EPSCoR (National Science Foundation). Y.C. acknowledges support from the NDSU startup funds, the NSF EPSCoR New Faculty Award, and the ND NASA EPSCoR RID Grant. TEM material is based upon work supported by the National Science Foundation under Grant No. 0923354. Any opinions, findings, and conclusions or recommendations expressed in this material are those of the author(s) and do not necessarily reflect the views of the National Science Foundation.

\section{References}

1. Helmlinger G, Yuan F, Dellian M, Jain RK. Interstitial pH and pO2 gradients in solid tumors in vivo: high-resolution measurements reveal a lack of correlation. Nat. Med. 1997; 3(2):177-182. [PubMed: 9018236]

2. Vaupel P, Schlenger K, Knoop C, Höckel M. Oxygenation of human tumors: evaluation of tissue oxygen distribution in breast cancers by computerized O2 tension measurements. Cancer Res. 1991; 51(12):3316-3322. [PubMed: 2040005]

3. Carmeliet P, Jain RK. Angiogenesis in cancer and other diseases. Nature. 2000; 407(6801):249-257. [PubMed: 11001068] 
4. Semenza GL. Hypoxia and cancer. Cancer Metastasis Rev. 2007; 26(2):223. [PubMed: 17404692]

5. Zhong H, De Marzo AM, Laughner E, Lim M, Hilton DA, Zagzag D, Buechler P, Isaacs WB, Semenza GL, Simons JW. Overexpression of hypoxia-inducible factor 1a in common human cancers and their metastases. Cancer Res. 1999; 59(22):5830-5835. [PubMed: 10582706]

6. Duffy JP, Eibl G, Reber HA, Hines OJ. Influence of hypoxia and neoangiogenesis on the growth of pancreatic cancer. Mol. Cancer. 2003; 2(1):12. [PubMed: 12605718]

7. Li D, Xie K, Wolff R, Abbruzzese JL. Pancreatic cancer. Lancet. 2004; 363(9414):1049-1057. [PubMed: 15051286]

8. Moore MJ, Goldstein D, Hamm J, Figer A, Hecht JR, Gallinger S, Au HJ, Murawa P, Walde D, Wolff RA, et al. Erlotinib plus gemcitabine compared with gemcitabine alone in patients with advanced pancreatic cancer: a phase III trial of the National Cancer Institute of Canada Clinical Trials Group. J. Clin. Oncol. 2007; 25(15):1960-1966. [PubMed: 17452677]

9. Brown JM, Wilson WR. Exploiting tumour hypoxia in cancer treatment. Nat. Rev. Cancer. 2004; 4(6):437-447. [PubMed: 15170446]

10. Meng F, Zhong Z, Feijen J. Stimuli-responsive polymersomes for programmed drug delivery. Biomacromolecules. 2009; 10(2):197-209. [PubMed: 19123775]

11. Stuart MAC, Huck WT, Genzer J, Müller M, Ober C, Stamm M, Sukhorukov GB, Szleifer I, Tsukruk VV, Urban M, et al. Emerging applications of stimuli-responsive polymer materials. Nat. Mater. 2010; 9(2):101-113. [PubMed: 20094081]

12. Thambi T, Deepagan V, Yoon HY, Han HS, Kim S-H, Son S, Jo D-G, Ahn C-H, Suh YD, Kim K, et al. Hypoxia-responsive polymeric nanoparticles for tumor-targeted drug delivery. Biomaterials. 2014; 35(5):1735-1743. [PubMed: 24290696]

13. Perche F, Biswas S, Wang T, Zhu L, Torchilin V. Hypoxia-Targeted siRNA Delivery. Angew. Chem. 2014; 126(13):3430-3434.

14. Kiyose K, Hanaoka K, Oushiki D, Nakamura T, Kajimura M, Suematsu M, Nishimatsu H, Yamane T, Terai T, Hirata Y, Nagano T. Hypoxia-sensitive fluorescent probes for in vivo real-time fluorescence imaging of acute ischemia. J. Am. Chem. Soc. 2010; 132(45):15846-15848. [PubMed: 20979363]

15. Burris, Hr, Moore, MJ., Andersen, J., Green, MR., Rothenberg, ML., Modiano, MR., Cripps, MC., Portenoy, RK., Storniolo, AM., Tarassoff, P. Improvements in survival and clinical benefit with gemcitabine as first-line therapy for patients with advanced pancreas cancer: a randomized trial. J. Clin. Oncol. 1997; 15(6):2403-2413. [PubMed: 9196156]

16. Ciardiello F, Tortora G. EGFR antagonists in cancer treatment. N. Engl. J. Med. 2008; 358(11): 1160-1174. [PubMed: 18337605]

17. Kamath S, Kummerow F, Narayan KA. A simple procedure for the isolation of rat liver microsomes. FEBS Lett. 1971; 17(1):90-92. [PubMed: 11946004]

18. Letchford K, Burt H. A review of the formation and classification of amphiphilic block copolymer nanoparticulate structures: micelles, nanospheres, nanocapsules and polymersomes. Eur. J. Pharm. Biopharm. 2007; 65(3):259-269. [PubMed: 17196803]

19. Perche F, Biswas S, Wang T, Zhu L, Torchilin V. Hypoxia-Targeted siRNA Delivery. Angew. Chem. Int. Ed. 2014; 53(13):3362-3366.

20. Chevalier A, Piao W, Hanaoka K, Nagano T, Renard P-Y, Romieu A. Azobenzene-caged sulforhodamine dyes: a novel class of 'turn-on' reactive probes for hypoxic tumor cell imaging. Methods Appl. Fluoresc. 2015; 3(4):044004.

21. Gabizon A, Catane R, Uziely B, Kaufman B, Safra T, Cohen R, Martin F, Huang A, Barenholz Y. Prolonged circulation time and enhanced accumulation in malignant exudates of doxorubicin encapsulated in polyethylene-glycol coated liposomes. Cancer Res. 1994; 54(4):987-992. [PubMed: 8313389]

22. Discher BM, Won Y-Y, Ege DS, Lee JC, Bates FS, Discher DE, Hammer DA. Polymersomes: tough vesicles made from diblock copolymers. Science. 1999; 284(5417):1143-1146. [PubMed: 10325219]

23. Du Y, Chen W, Zheng M, Meng F, Zhong Z. pH-sensitive degradable chimaeric polymersomes for the intracellular release of doxorubicin hydrochloride. Biomaterials. 2012; 33(29):7291-7299. [PubMed: 22795540] 
24. Kim MS, Lee DS. Biodegradable and $\mathrm{pH}$-sensitive polymersome with tuning permeable membrane for drug delivery carrier. Chem. Commun. 2010; 46(25):4481-4483.

25. Howse JR, Jones RA, Battaglia G, Ducker RE, Leggett GJ, Ryan AJ. Templated formation of giant polymer vesicles with controlled size distributions. Nat. Mater. 2009; 8(6):507-511. [PubMed: 19448615]

26. Kulkarni PS, Haldar MK, Nahire RR, Katti P, Ambre AH, Muhonen WW, Shabb JB, Padi SK, Singh RK, Borowicz PP, et al. MMP-9 Responsive PEG cleavable nanovesicles for efficient delivery of chemotherapeutics to pancreatic cancer. Mol. Pharmaceutics. 2014; 11(7):2390-2399.

27. Justice BA, Badr NA, Felder RA. 3D cell culture opens new dimensions in cell-based assays. Drug Discovery Today. 2009; 14(1):102-107. [PubMed: 19049902]

28. Lee J, Lilly GD, Doty RC, Podsiadlo P, Kotov NA. In vitro toxicity testing of nanoparticles in 3D cell culture. Small. 2009; 5(10):1213-1221. [PubMed: 19263430]

29. Yoshii Y, Waki A, Yoshida K, Kakezuka A, Kobayashi M, Namiki H, Kuroda Y, Kiyono Y, Yoshii $\mathrm{H}$, Furukawa T, et al. The use of nanoimprinted scaffolds as 3D culture models to facilitate spontaneous tumor cell migration and well-regulated spheroid formation. Biomaterials. 2011; 32(26):6052-6058. [PubMed: 21640378]

30. Durand RE, Olive PL. Evaluation of bioreductive drugs in multicell spheroids. Int. J. Radiat. Oncol. Biol., Phys. 1992; 22(4):689-692. [PubMed: 1544838] 

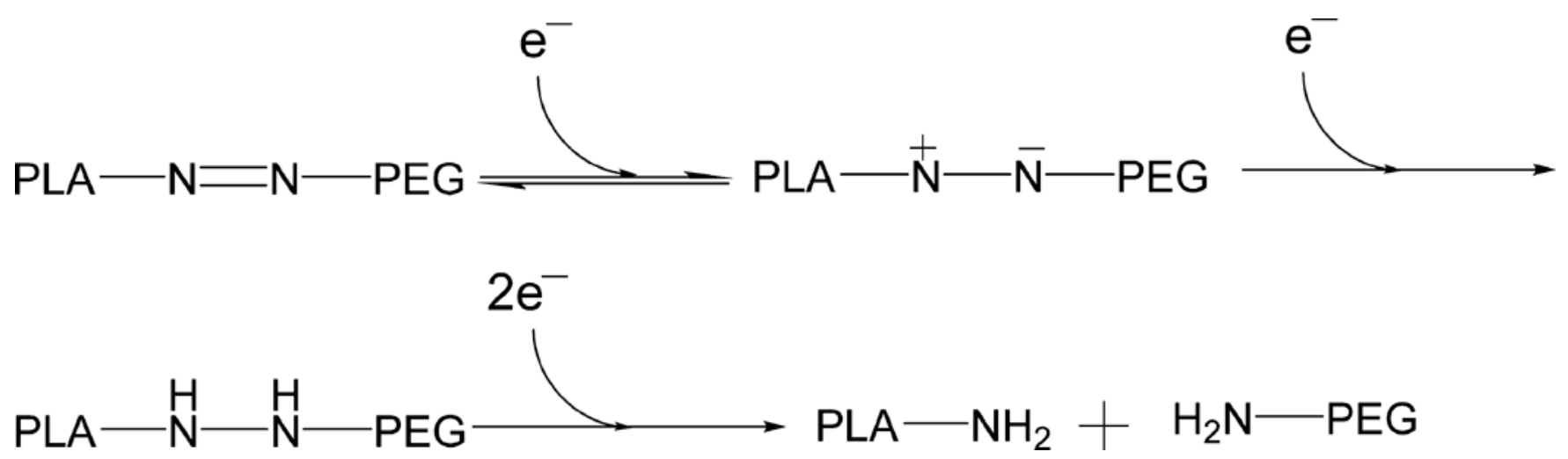

Figure 1.

Proposed mechanism of azobenzene reduction in hypoxic, reducing environment. ${ }^{14}$ 

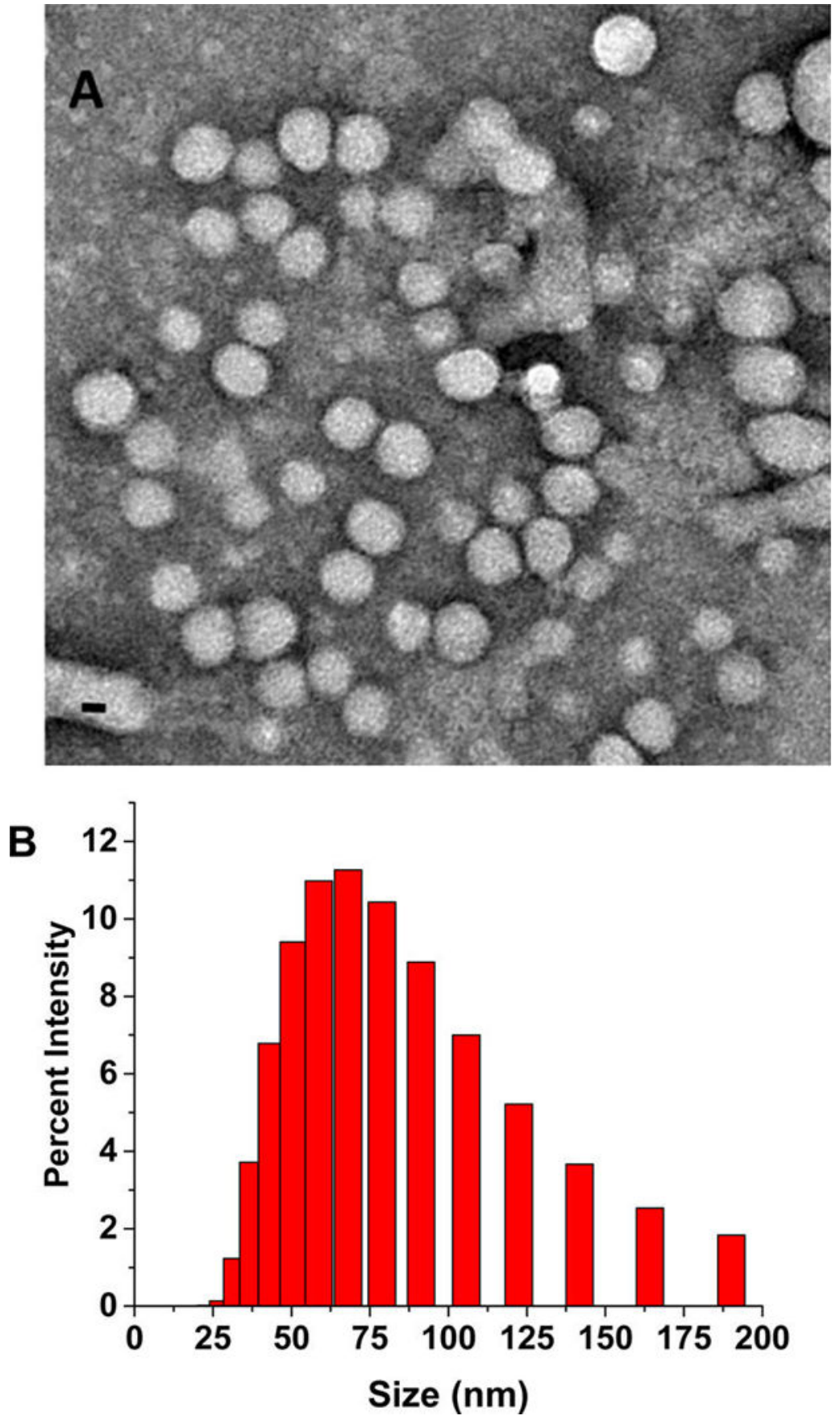

Figure 2.

Transmission electron microscopic (TEM) image of polymersomes (scale bar, $20 \mathrm{~nm}$ ) (A) and the size distribution profile by DLS (B). 


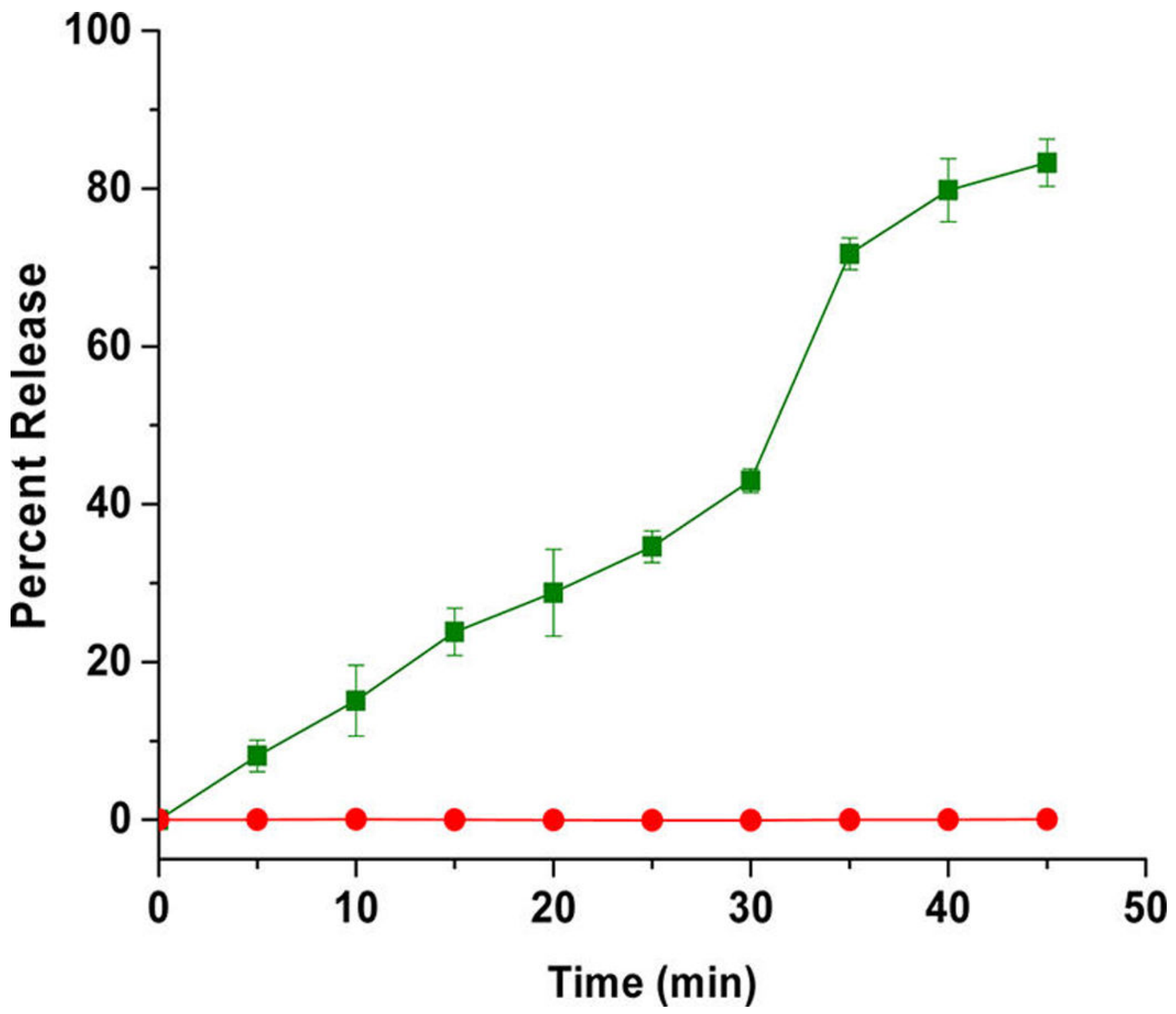

Figure 3.

Cumulative release of encapsulated carboxyfluorescein from polymersomes under normoxic (red circles) and hypoxic conditions (green squares). The lines connecting the data points are also shown $(N=3)$. 


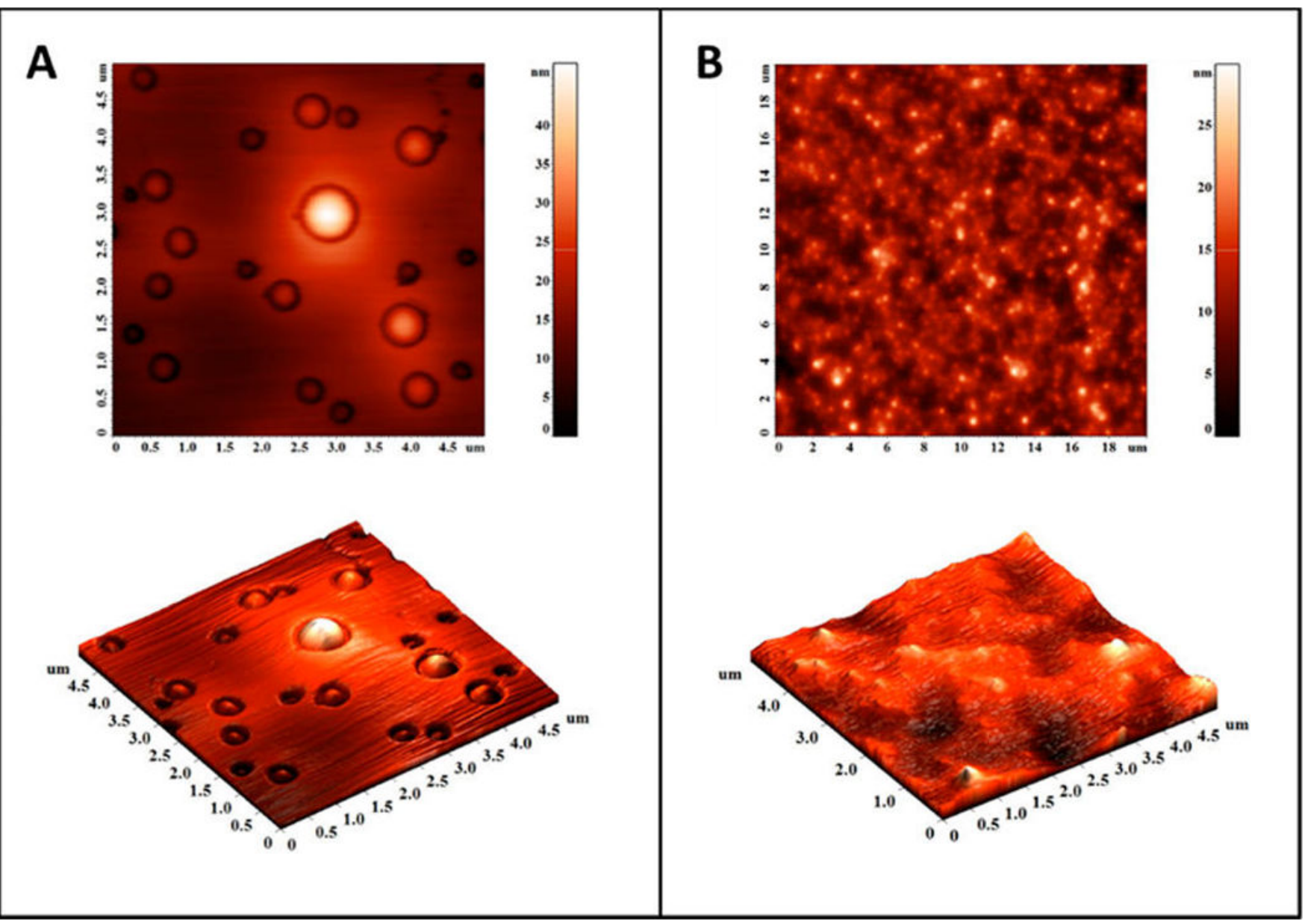

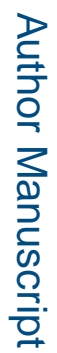

Figure 4.

Atomic force microscopic images of the polymersomes under normoxic (A) and hypoxic conditions (B). 

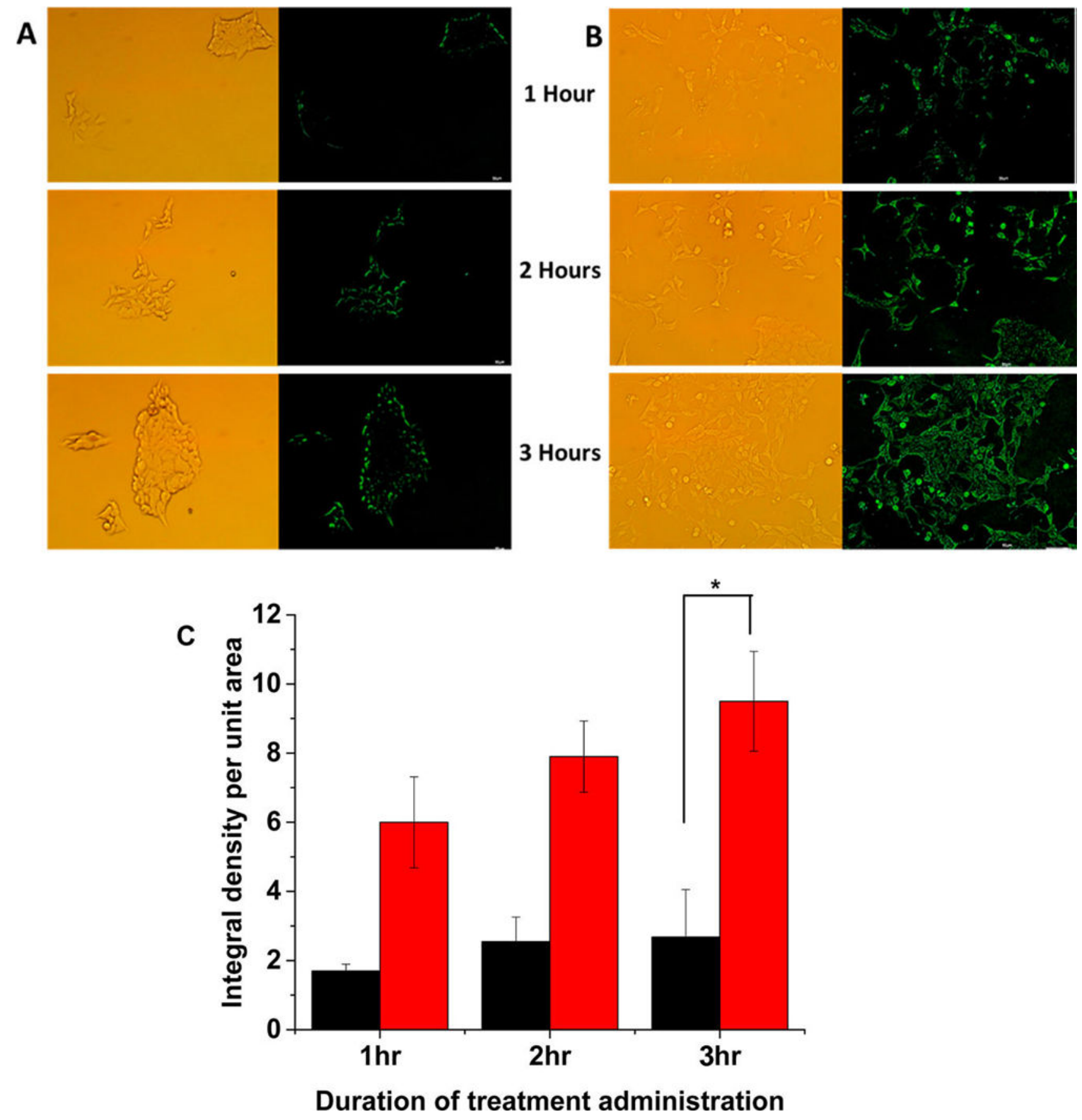

Figure 5.

Confocal fluorescence microscopic images of the BxPC-3 cells incubated with carboxyfluorescein-encapsulated polymersomes under normoxic (A) and hypoxic (B) conditions for 1, 2, and $3 \mathrm{~h}$. (C) Quantitative fluorescence integral density for the images shown in panels A and B indicating uptake in cells cultured under normoxic (black) and hypoxic (red) conditions $(N=3, * p<0.05)$. 


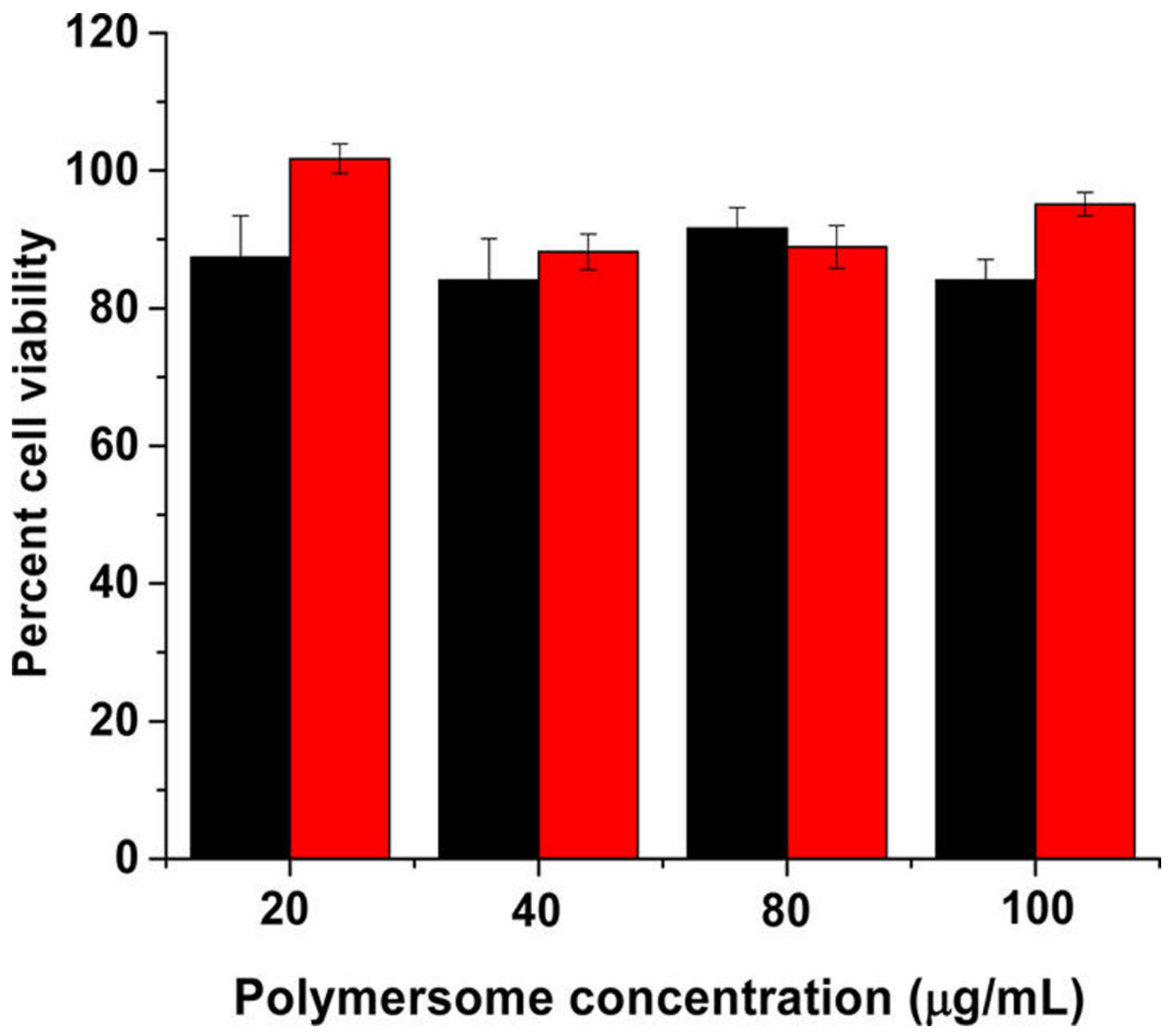

Figure 6.

Viability of the BxPC-3 cells with polymersomes encapsulating phosphate buffer $(\mathrm{pH}=7.4)$ under hypoxic (black bars) and normoxic (red bar) conditions $(N=4)$. 

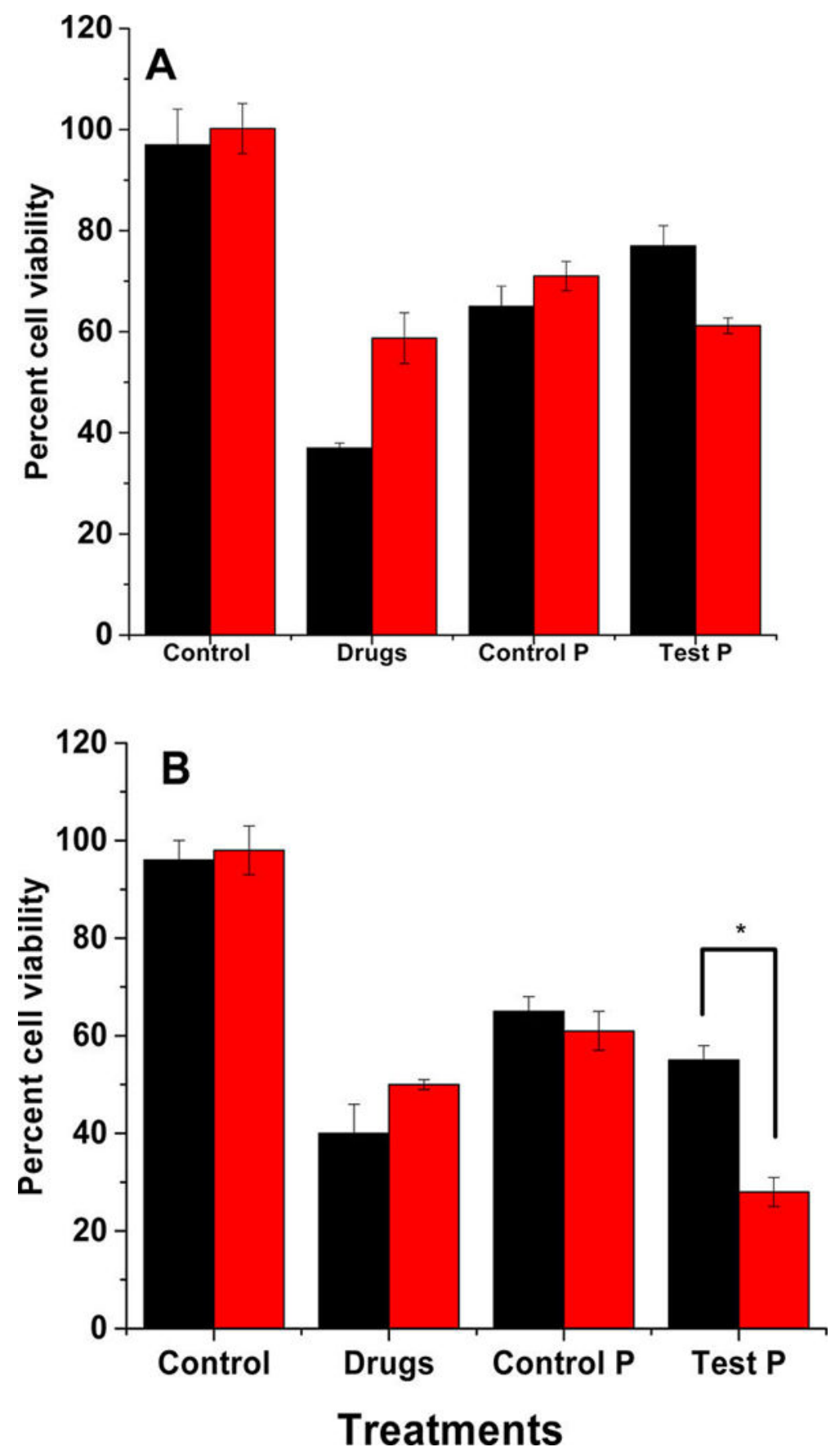

Figure 7.

Viability of the BxPC-3 cells in monolayer (A) and spheroidal (B) cultures after treatment with the control (hypoxia-responsive polymersomes without any drugs), anticancer drugs (Drugs), drug encapsulated polymersomes prepared from PLLA $5000-\mathrm{PEG}_{2000}$ (Control P), and the hypoxia-responsive vesicles (Test P) under normoxic (black bars) and hypoxic (red bars) conditions $(N=6, * P<0.05)$. 
$\mathrm{HO}$<smiles>COCC(C)OCC(C)(C)NC(=O)c1ccc(N=Nc2ccc(C(=O)O)cc2)cc1</smiles>

(1) $\mathrm{H}_{2} \mathrm{~N} \curvearrowright \mathrm{OH}$

Pyridine, NHS, EDC (83\%)

(2) D,L-lactide dioctyl tin oxide (catalytic) $\checkmark(67 \%)$<smiles>C=C(OC(=O)C(C)(C)O)C(=O)OCCCNC(=O)c1ccc(N=Nc2ccc(C(=O)NC(C)COCCOC)cc2)cc1</smiles>

Scheme 1.

Synthesis of the Azobenzene Incorporated, Hypoxia-Responsive Polymer ${ }^{\mathrm{a}}$

${ }^{a}$ The hypoxia-responsive unit is shown in red. 
Table 1

Hydrodynamic Diameters and the PDI for Polymersome Formulations Determined by DLS

\begin{tabular}{lcc}
\hline formulation & $\begin{array}{c}\text { average diameter } \\
(\mathbf{n m}) \pm \text { SD }\end{array}$ & PDI \pm SD \\
\hline buffer encapsulated polymersomes & $83.6 \pm 1.9$ & $0.30 \pm 0.02$ \\
carboxyfluorescein encapsulated polymersomes & $136.4 \pm 1.1$ & $0.25 \pm 0.03$ \\
drug encapsulated polymersomes & $262.6 \pm 30$ & $0.35 \pm 0.06$ \\
\hline
\end{tabular}

